\title{
Reconhecimento dos Revisores "ad hoc" ativos durante 0 ano de 2010
}

\author{
Recognition of active "ad hoc" reviewers during the year 2010
}

Wallace Chamon

Wallace Chamon

\section{REVISORES “AD HOC” ATIVOS DURANTE O ANO DE 2010:}

Acácio Alves de Souza Lima Filho

Adriana Santos Forseto

Amélia Kamegasawa

Ana Estela Besteti Pires Ponce Sant'Anna

André Okanobo

Andréia Peltier Urbano

Angelino Júlio Cariello

Augusto de Queirós Duarte

Bruno Castelo Branco

Carlos Alexandre de Amorim Garcia Filho

Carlos Eduardo Leite Arieta

Célia Simões C. de Oliveira Sathler

Cintia Sade de Paiva

Cláudia Shiratori de Oliveira

Consuelo Bueno Diniz Adan

Denise de Vuono Chinzon

Denise Fornazari de Oliveira

Diane Ruschel Marinho

Edison João Geraissate Filho

Edmea Rita Temporini

Edson Procianoy

Eduardo Buchelle Rodrigues

Eduardo Cunha de Souza

Eric Pinheiro de Andrade

Fabiano Cade

Felício Aristóteles da Silva

Fernando Cançado Trindade
Fernando César Abib

Flávia Augusta Attié de Castro

Flávio Eduardo Hirai

Francisco Assis Cordeiro Barbosa

Francisco Eduardo Lopes de Lima

Frederico Castelo Moura

Gustavo Castro de Oliveira

Helia Soares Angotti

Hélio Angotti-Neto

Italo Mundialino Marcon

Jackson Barreto Júnior

João Antonio Prata Júnior

Joaquim Pereira Paes

José Byron Vicente Dias Fernandes

Joyce Hisae Yamamoto

Juliana Maria Ferraz Sallum

Leonardo Provetti Cunha

Leopoldo Magacho

Lineu Shiroma

Lisandro Massanori Sakata

Luciane Bugman Moreira

Luciene Barbosa de Sousa

Luís Alberto Carvalho

Luís Antonio Vieira

Luís Carlos Ferreira de Sá

Luís Eduardo Morato Rebouças de Carvalho

Luiz Carlos Molinari Gomes 
Luiz Henrique Soares Gonçalves Lima

Luiz Vicente Rizzo

Magno Antonio Ferreira

Marcelo Hatanaka

Marcos Carvalho da Cunha

Maria de Lourdes Motta Moreira Villas Boas

Maria Emília Xavier dos Santos Araujo

Marisa Zamora Kattah

Mariza Aparecida Polati

Maurício Bastos Pereira

Maurício Della Paolera

Mauro Nishi

Milton Nunes de Moraes Filho

Milton Yogi

Mirian Skaf

Mirko Jankov

Mônica de C. Alves Paula

Nelson Alexandre Sabrosa

Niro Kasahara

Núbia Cristina Freitas Maia

Patrícia Grativol Costa

Patrícia Mitiko Santello Akaishi

Paulo Gilberto Jorge Fadel

Paulo Pierre Filho
Paulo Sérgio Horta Barbosa

Pedro Carricondo

Ramon Ghanem

Ricardo Morschbacher

Ricardo Suzuki

Ricardo Themudo Lessa Waetge

Roberto Freda

Roberto Lauande Pimentel

Roberto Mitiaki Endo

Roberto Murillo Limongi de Souza Carvalho

Ronaldo Boaventura Barcellos

Rosa Maria Graziano

Rubens Belfort Neto

Ruth Rosenhek Schor

Samuel Rymer

Sandra Maria Canelas Beer

Sebastião Cronemberger

Seiji Hayashi

Sérgio Vanetti Burnier

Sérgio Henrique Teixeira

Solange Rios Salomão

Tânia Pereira Nunes

Vinicius Coral Ghanem

Wesley Ribeiro Campos 